\title{
ATLAS Results on Heavy Flavor Production and Decays
}

\author{
Sally Seidel ${ }^{a, *}$, for the ATLAS Collaboration \\ a Department of Physics and Astronomy, University of New Mexico, \\ 210 Yale Blvd. NE, Albuquerque, NM 87106, USA \\ E-mail: sally.seidelecern.ch
}

Heavy flavor production and decays are studied with the ATLAS detector at the Large Hadron Collider, mainly through final states containing muons. A measurement of the $B_{c}{ }^{ \pm}$meson production cross section relative to that of the $B^{ \pm}$meson is presented. The results of a study of flavor-changing neutral current processes in the decays of $B_{s}$ and $B^{0}$ into two muons are also described. 


\section{Introduction}

Heavy flavor production and decays are studied with the ATLAS detector [1] at the Large Hadron Collider (LHC), mainly through final states containing muons. A measurement of the $B_{c}{ }^{ \pm}$meson production cross section relative to that of the $B^{ \pm}$meson is presented. The results of a study of flavor-changing neutral current processes in the decays of $B_{s}{ }^{0}$ and $B^{0}$ into two muons are also described.

\section{Measurement of the Relative $B_{c}^{ \pm} / B^{ \pm}$Production Cross Section at $\sqrt{s}_{\mathbf{s}}=8 \mathrm{TeV}$}

\subsection{Overview}

The total and differential cross sections for the production of $B_{c}{ }^{ \pm}$mesons times their branching fraction to $J / \psi \pi^{ \pm}$are measured [2] relative to those for the production of $B^{ \pm}$mesons times their branching fraction to $J / \psi K^{ \pm}$. The data used for this study correspond to an integrated luminosity of $20.3 \mathrm{fb}^{-1}$ of pp collisions recorded by the ATLAS detector in 2012 at a center-of-mass energy of $\sqrt{\mathrm{s}}_{\mathrm{s}}=8 \mathrm{TeV}$. The measurement is performed differentially in bins of transverse momentum $\mathrm{p}_{\mathrm{T}}$ for $13 \mathrm{GeV}<\mathrm{p}_{\mathrm{T}}\left(B_{c}{ }^{ \pm}\right)<22 \mathrm{GeV}$ and $\mathrm{p}_{\mathrm{T}}\left(B_{c}{ }^{ \pm}\right)>22 \mathrm{GeV}$ and in bins of rapidity $\mathrm{y}$ for $|\mathrm{y}|<0.75$ and $0.75<|\mathrm{y}|<2.3$. The bins are selected to equalize yields of the $B_{c}{ }^{ \pm}$signal, and the same bin sizes are used for the $B_{c}{ }^{ \pm}$and $B^{ \pm}$mesons. The relative cross section times branching fraction for the full range $\mathrm{p}_{\mathrm{T}}>13 \mathrm{GeV}$ and $|\mathrm{y}|<2.3$ is also measured, and the result is $\left(0.34 \pm 0.04_{\text {stat }} \pm 0.02_{\text {sys }} \pm\right.$ $\left.0.01_{\text {lifetime }}\right) \%$.

\subsection{Details of the Measurement}

The $B_{c}{ }^{ \pm}$meson is a bound state of the two heaviest distinct quarks able to form a stable state, $c$ and $\bar{b}$ for $B_{c}{ }^{+}$or $\bar{c}$ and $b$ for $B_{c}^{-}$. Measurements of its production can provide unique insight into heavy-quark hadronization: unlike lighter $B$-states, production of a $B_{c}{ }^{ \pm}$meson requires collinear production of two distinct heavy quarks.

The $J / \psi$ candidates are reconstructed from pairs of oppositely charged muon candidates. The muon mass is assigned to each track in the $J / \psi$ reconstruction. The re-fitted track parameters are derived from the vertex fit. The $B$ candidates (where $B$ represents $B_{c}{ }^{ \pm}$or $B^{ \pm}$) are reconstructed by fitting the tracks of the two muons from the $J / \psi$ candidate, together with a charged-hadron track, to a common vertex. For the $B^{ \pm}$candidates, the kaon mass is assigned to the hadron track, while for the $B_{c}{ }^{ \pm}$candidates, the pion mass is assigned to the hadron track. The mass of the $J / \psi$ meson is constrained in the fit to its world average value. The element of the combinatorial background that consists of $J / \psi$ mesons combined with light hadrons that are not associated with the decay is reduced by requiring a minimum value for the significance of the impact parameter of the hadron track relative to the primary vertex in the transverse plane. The background due to partially reconstructed semi-leptonic decays of the $B_{c}{ }^{ \pm}$is reduced by removing combinations in which one of the hadronic candidates is identified as a muon by the ATLAS muon spectrometer. Reconstructed $B$ candidates are required to satisfy the following selection criteria: (1) the $\chi^{2} /\left(\mathrm{N}_{\text {d.o.f. }}=4\right)$ of the fit of the $B$ vertex must be below 1.8; (2) the rapidity $|\mathrm{y}(B)|$ must be less than 2.3 ; (3) the $\mathrm{p}_{\mathrm{T}}$ of the hadron candidate must be above $2.0 \mathrm{GeV}$; and (4) the impact parameter significance of the hadron candidate must exceed 1.2. In events with multiple $B$ candidates, the candidate with the best $\chi^{2}$ from the vertex fits is used.

The relative cross section times branching fraction is given by: 


$$
\frac{\sigma\left(B_{c}^{ \pm}\right) \cdot B\left(B_{c}^{ \pm} \rightarrow J / \psi \pi^{ \pm}\right) \cdot B\left(J / \psi \rightarrow \mu^{+} \mu^{-}\right)}{\sigma\left(B^{ \pm}\right) \cdot B\left(B^{ \pm} \rightarrow J / \psi K^{ \pm}\right) \cdot B\left(J / \psi \rightarrow \mu^{+} \mu^{-}\right)}=\frac{N^{\text {reco }}\left(B_{c}^{ \pm}\right)}{N^{\text {reco }}\left(B^{ \pm}\right)} \cdot \frac{\varepsilon\left(B^{ \pm}\right)}{\varepsilon\left(B_{c}^{ \pm}\right)} .
$$

The notation $N^{\text {reco }}(X)$ refers to the number of reconstructed collision data events, where $X$ is either $B_{c}{ }^{ \pm}$or $B^{ \pm}$. The $\varepsilon\left(B^{ \pm}\right)$and $\varepsilon\left(B_{c}{ }^{ \pm}\right)$are the efficiencies of $B^{ \pm}$and $B_{c}{ }^{ \pm}$reconstruction that correct the numbers $N^{\text {reco }}\left(B^{ \pm}\right)$and $N^{\text {reco }}\left(B_{c}^{ \pm}\right)$for detector effects, selection criteria, differences between interactions of $K^{ \pm}$and $\pi^{ \pm}$with the detector material, as well as efficiencies associated with the trigger.

Extended unbinned maximum-likelihood fits to the mass distributions of the $B_{c}{ }^{ \pm}$and the $B^{ \pm}$are performed to extract $N^{\text {reco }}\left(B_{c}^{ \pm}\right)$and $N^{\text {reco }}\left(B^{ \pm}\right)$from the data in each bin in $\mathrm{p}_{\mathrm{T}}$ and $|\mathrm{y}|$. This involves calculating the parameters that maximize a likelihood function that includes the signal contribution, partially reconstructed $b$-hadron decays, the Cabibbo-suppressed decay $B^{ \pm} \rightarrow J / \psi \pi^{ \pm}$, and the remaining background which is mostly due to production of $J / \psi$ mesons from decays of $b$ hadrons other than the $B^{ \pm}$, which are then combined with a random hadron track. The efficiencies for this measurement encompass those of the $J / \psi$ trigger, the muon spectrometer, and the inner detector, the efficiency of fitting the muon and hadron tracks to a common vertex, and the efficiency of the selection criteria. Systematic uncertainties are associated with the size of the Monte Carlo (MC) samples used, the Monte Carlo reweighting procedure, the application of minimal selection criteria, the tracking uncertainty, the choice of signal model in the fit, the model for the Cabibbo-suppressed decay, the background model, trigger and reconstruction effects, and the $B$ meson lifetime.

Figure 1 summarizes the measurement. The differential relative production cross section for the inclusive selection containing all events in the range $\mathrm{p}_{\mathrm{T}}>13 \mathrm{GeV}$ and $|\mathrm{y}|<2.3$ is $\left(0.34 \pm 0.04_{\text {stat }}\right.$ $\pm 0.02_{\text {syst }} \pm 0.01_{\text {lifetime }} \% \%$. Results for individual bins are:

- for $13 \mathrm{GeV}<\mathrm{p}_{\mathrm{T}}<22 \mathrm{GeV},|\mathrm{y}|<2.3:\left(0.44 \pm 0.07_{\text {stat }} \pm 0.04_{\text {syst }} \pm 0.01_{\text {lifetime }}\right) \%$,

- for $\mathrm{p}_{\mathrm{T}}>22 \mathrm{GeV},|\mathrm{y}|<2.3:\left(0.24 \pm 0.04_{\text {stat }} \pm 0.01_{\text {syst }} \pm 0.01_{\text {lifetime }}\right) \%$,

- $\quad$ for $\mathrm{p}_{\mathrm{T}}>13 \mathrm{GeV},|\mathrm{y}|<0.75:\left(0.38 \pm 0.06_{\text {stat }} \pm 0.04_{\text {syst }} \pm 0.01_{\text {lifetime }}\right) \%$,

- for $\mathrm{p}_{\mathrm{T}}>13 \mathrm{GeV}, 0.75<|\mathrm{y}|<2.3:\left(0.29 \pm 0.05_{\text {stat }} \pm 0.02_{\text {syst }} \pm 0.01_{\text {lifetime }}\right) \%$.
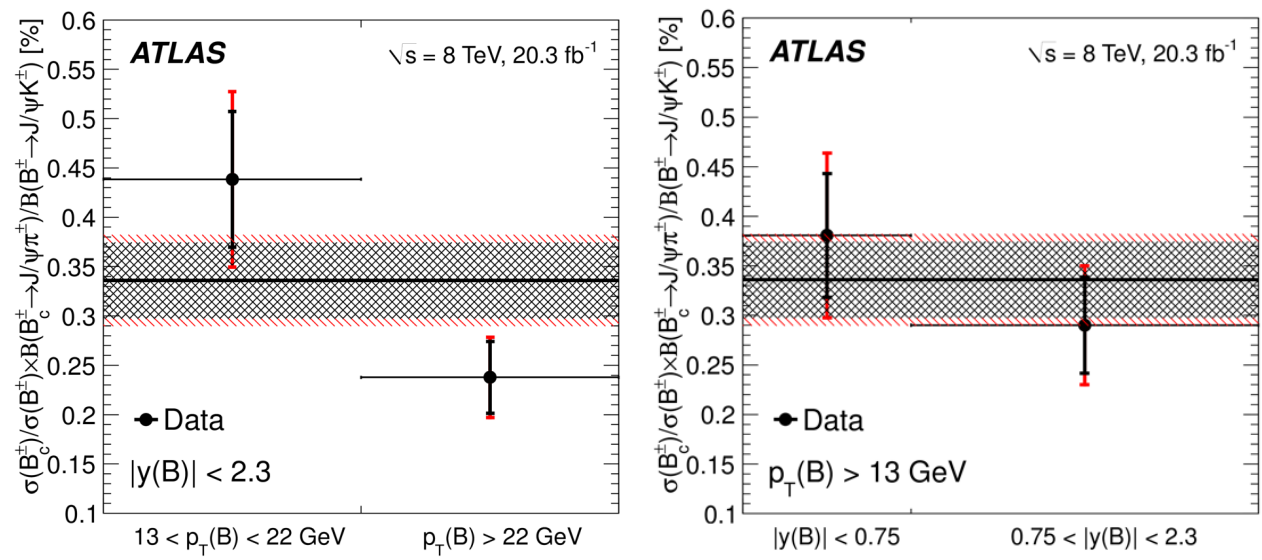

Figure 1. Summary of the cross section measurement. The left figure shows the production cross section for the $B_{c}{ }^{ \pm}$relative to the $B^{ \pm}$(times the corresponding branching fractions) for two bins in p (black data points) and for the inclusive bin (horizontal band). The right figure shows the measurement binned in rapidity (black data points). In each figure the horizontal bar indicates the bin width. The vertical error bars on the data points indicate the statistical uncertainty. The outer error bars indicate the size of the quadrature sum of uncertainties from all sources: statistical, systematic, and lifetime. The double hatched error band indicates the statistical uncertainty, while the single hatched bands indicate the quadrature sum of uncertainties from all sources. Figure source: [2]. 
The differential measurement suggests a dependence on the transverse momentum: the production cross section of the $B_{c}{ }^{ \pm}$decreases faster with $\mathrm{p}_{\mathrm{T}}$ than the production cross section of the $B^{ \pm}$. No significant dependence on rapidity is observed.

\section{Study of the Rare Decays of $B_{s}{ }^{0}$ and $B^{0}$ Mesons into Muon Pairs Using Data Collected During 2015 and 2016}

\subsection{Overview}

A study of the decays $B_{s}{ }^{0} \rightarrow \mu^{+} \mu$ and $B^{0} \rightarrow \mu^{+} \mu$ has been performed [3] using $26.3 \mathrm{fb}^{-1}$ of $13 \mathrm{TeV}$ LHC proton-proton collision data collected with the ATLAS detector in 2015 and 2016. Since the detector resolution in $\mu^{+} \mu$ invariant mass is comparable to the $B_{s}{ }^{0}-B^{0}$ mass difference, a single fit determines the signal yields for both decay modes. This results in a measurement of the branching fraction $B\left(B_{s}^{0} \rightarrow \mu^{+} \mu^{-}\right)=\left(3.2_{-1.0}^{+1.1}\right) \times 10^{-9}$ and an upper limit $B\left(B^{0} \rightarrow \mu^{+} \mu^{-}\right)<4.3 \times 10^{-10}$ at $95 \%$ confidence level. The measurement is combined with the Run 1 ATLAS result [4], yielding $B\left(B_{s}^{0} \rightarrow \mu^{+} \mu^{-}\right)=\left(2.8_{-0.7}^{+0.8}\right) \times 10^{-9}$ and an upper limit $B\left(B^{0} \rightarrow \mu^{+} \mu^{-}\right)<2.1 \times 10^{-10}$ at $95 \%$ confidence level. The combined result is consistent with the Standard Model (SM) prediction within 2.4 standard deviations in the $B\left(B^{0} \rightarrow \mu^{+} \mu\right)-B\left(B_{s}{ }^{0} \rightarrow \mu^{+} \mu^{-}\right)$plane.

\subsection{Details of the Measurement}

Flavor-changing neutral current processes are highly suppressed in the SM. The branching fractions of the decays $B_{(s)}^{0} \rightarrow \mu^{+} \mu^{-}$are, in addition, helicity suppressed in the SM, and are predicted to be:

$$
B\left(B_{s}^{0} \rightarrow \mu^{+} \mu^{-}\right)=(3.65 \pm 0.23) \times 10^{-9} \text { and } B\left(B^{0} \rightarrow \mu^{+} \mu^{-}\right)=(1.06 \pm 0.09) \times 10^{-10} .
$$

The smallness and precision of these predicted branching fractions provide a favorable environment for observing contributions from new physics: significant deviations from SM predictions could arise in models involving non-SM heavy particles, such as those predicted in the Minimal Supersymmetric Standard Model and in extensions such as Minimal Flavor Violation, TwoHiggs-Doublet Models, and others.

The $B_{s}{ }^{0} \rightarrow \mu^{+} \mu$ and $B^{0} \rightarrow \mu^{+} \mu^{-}$branching fractions are measured relative to the reference decay mode $B^{+} \rightarrow J / \psi\left(\rightarrow \mu^{+} \mu^{-}\right) K^{+}$which is abundant and has a well-measured branching fraction $B\left(B^{+} \rightarrow J / \psi K^{+}\right) \times B\left(J / \psi \rightarrow \mu^{+} \mu^{-}\right)$. The $B^{0} \rightarrow \mu^{+} \mu^{i}\left(B_{s}{ }^{0} \rightarrow \mu^{+} \mu^{-}\right)$branching fraction can be extracted as

$$
B\left(B_{(s)}^{0} \rightarrow \mu^{+} \mu^{-}\right)=\frac{N_{d(s)}}{\varepsilon_{\mu^{+} \mu^{-}}} \times\left[B\left(B^{+} \rightarrow J / \psi K^{+}\right) \times B\left(J / \psi \rightarrow \mu^{+} \mu^{-}\right)\right] \frac{\varepsilon_{J / \psi K^{+}}}{N_{J / \psi K^{+}}} \times \frac{f_{u}}{f_{d(s)}},
$$

where $N_{d}\left(N_{s}\right)$ is the $B^{0} \rightarrow \mu^{+} \mu^{-}\left(B_{s}{ }^{0} \rightarrow \mu^{+} \mu^{-}\right)$signal yield, $N_{J / \psi K+}$ is the $B^{+} \rightarrow J / \psi K^{+}$reference channel yield, $\varepsilon_{\mu^{+} \mu-}$ and $\varepsilon_{J / \psi K^{+}}$are the corresponding values of acceptance times efficiency measured in fiducial regions, and $f_{u} / f_{d}\left(f_{u} / f_{s}\right)$ is the ratio of the hadronization probabilities of a $b$-quark into $B^{+}$ and $B^{0}\left(B_{s}^{0}\right)$. Signal and reference channel events are selected with similar dimuon triggers. One half of the reference channel sample is used to determine the normalization and the other half is used to tune the kinematic distributions of simulated events.

A blind analysis was performed in which data in the dimuon invariant mass range were removed until the procedures for event selection and the details of signal yield extraction were completely defined. The final sample of candidates is selected using a multivariate classifier implemented as a boosted decision tree (BDT), designed to enhance the signal relative to the dominant dimuon 
background component. Checks on the distributions of the variables used in the multivariate classifier are based on the comparison of data and simulation for dimuon events, for $B^{+} \rightarrow J / \psi K^{+}$candidates and for events selected as $B_{s}^{0} \rightarrow J / \psi \phi \rightarrow \mu^{+} \mu^{-} K^{+} K^{-}$, which provide an additional validation of the procedures used in the analysis. The signal yield is obtained with an unbinned maximum-likelihood fit performed on the dimuon invariant mass distribution.

The BDT is based on 15 variables which can be classified into three groups: (a) $B$ meson variables, related to the reconstruction of the decay vertex and to the collinearity between the reconstructed $B$ meson momentum vector and the flight vector between the production and decay vertices; $(b)$ variables describing the muons that form the $B$ meson candidate; and (c) variables related to the rest of the event. The selection of the variables aims to maximize the discrimination power of the classifier without introducing significant dependence on the invariant mass of the muon pair.

The reference channel yield is extracted with an unbinned extended maximum-likelihood fit to the $J / \psi K^{+}$invariant mass distribution. The functional forms used to model both the signal and the backgrounds are obtained from studies of MC samples. All the yields are extracted from the fit to data, while the shape parameters are determined from a simultaneous fit to data and MC samples. Free parameters are introduced for the mass scale and mass resolution to accommodate data-MC differences. The fit to the $J / \psi K^{+}$invariant mass distribution for all $B^{+}$candidates includes four components: $B^{+} \rightarrow J / \psi K^{+}$decays, Cabibbo-suppressed $B^{+} \rightarrow J / \psi \pi^{+}$decays in the right tail of the main peak, partially reconstructed $B$ decays where one or more of the final-state particles are missing, and the non-resonant background composed mostly of $b \bar{b} \rightarrow J / \psi X$ decays.

An unbinned extended maximum-likelihood fit is performed on the dimuon invariant mass distribution simultaneously across four BDT intervals, each including models for the respective signal and background contributions. The signal and background models are derived from simulations and from data collected in the mass sidebands of the search region. The invariant mass distribution of the $B_{(s)}{ }^{0} \rightarrow \mu^{+} \mu$ signal is described with two double-Gaussian distributions, centered respectively at the $B^{0}$ or $B_{s}{ }^{0}$ mass. The numbers of background events contained in the signal region $(5166-5526 \mathrm{MeV})$ are computed from the interpolation of the data observed in the sidebands. The branching fractions for the decays $B^{0} \rightarrow \mu^{+} \mu^{i}$ and $B_{s}{ }^{0} \rightarrow \mu^{+} \mu$ are extracted from data using a maximum-likelihood fit. The likelihood is obtained from the one used for $N_{s}$ and $N_{d}$ by replacing the fit parameters with the corresponding branching fractions divided by normalization terms, and including Gaussian multiplicative factors for the normalization uncertainties. The values of the branching fractions that maximize the profile likelihood are $B\left(B_{s}^{0} \rightarrow \mu^{+} \mu^{-}\right)=(3.2 \pm 0.9) \times 10^{-9}$ and $B\left(B^{0} \rightarrow \mu^{+} \mu^{-}\right)=(-1.3 \pm 2.1) \times 10^{-10}$. The likelihood function from the current result is combined with the likelihood function from the Run 1 result [4]. The combined likelihood maximum corresponds to $B\left(B_{s}^{0} \rightarrow \mu^{+} \mu^{-}\right)=(2.8 \pm 0.7) \times 10^{-9}, B\left(B^{0} \rightarrow \mu^{+} \mu^{-}\right)=(-1.9 \pm 1.6) \times 10^{-10}$.

When applying a one-dimensional Neyman construction to this combined likelihood, whose maximum is unconstrained and allowed to access the unphysical (negative) region, the $68.3 \%$ confidence interval is obtained as $B\left(B_{s}^{0} \rightarrow \mu^{+} \mu^{-}\right)=\left(2.8_{-0.7}^{+0.8}\right) \times 10^{-9}$ while the upper limit at $95 \%$ confidence level, determined with the same Neyman procedure, yields $B\left(B^{0} \rightarrow \mu^{+} \mu^{-}\right)<2.1 \times 10^{-10}$. Figure 2 shows the likelihood contours for the combined Run 1 and Run 2 results. The combined branching fraction measurements differ by 2.4 standard deviations from the SM values in the $B\left(B^{0} \rightarrow \mu^{+} \mu^{-}\right)-B\left(B_{s}^{0} \rightarrow \mu^{+} \mu^{-}\right)$plane. 


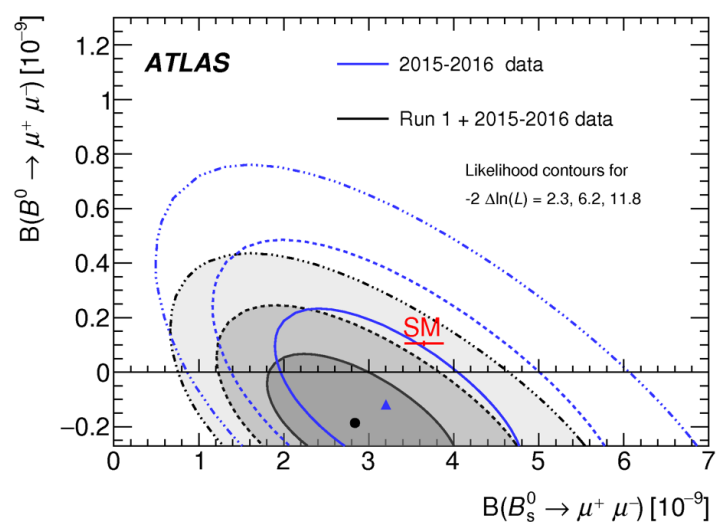

Figure 2. Likelihood contours for the combination of the Run 1 and 2015-2016 Run 2 results (shaded areas). The contours are obtained from the combined likelihoods of the two analyses, for values of $-2 \Delta \ln (L)$ equal to $2.3,6.2$, and 11.8. The empty contours represent the result from 2015 - 2016 Run 2 data alone. The Standard Model prediction with uncertainties is indicated. Figure source: [3].

\section{Conclusion}

The production cross section for the $B_{c}{ }^{ \pm}$meson relative to that of the $B^{ \pm}$meson, times their branching fractions to $J / \psi \pi^{ \pm}$, for the inclusive selection containing all events in the range $\mathrm{p}_{\mathrm{T}}>13 \mathrm{GeV}$ and $|\mathrm{y}|<2.3$ has been found to be $\left(0.34 \pm 0.04_{\text {stat }} \pm 0.02_{\text {syst }} \pm 0.01_{\text {lifetime }}\right) \%$. Results for differential cross sections in individual bins in $\mathrm{p}_{\mathrm{T}}$ and $|\mathrm{y}|$ suggest a dependence on the transverse momentum; no significant dependence on rapidity is observed. Application of a one-dimensional Neyman construction to the combined likelihood for the measurement of the branching fractions for the decays $B_{s}^{0} \rightarrow \mu^{+} \mu^{-}$and $B^{0} \rightarrow \mu^{+} \mu^{-}$indicates that this differs by 2.4 standard deviations from the SM values in the $B\left(B^{0} \rightarrow \mu^{+} \mu^{-}\right)-B\left(B_{s}^{0} \rightarrow \mu^{+} \mu^{-}\right)$plane.

\section{References}

[1] ATLAS Collaboration, "The ATLAS Detector at the CERN Large Hadron Collider," JINST 3 S08003 (2008).

[2] ATLAS Collaboration, "Measurement of the relative $B_{c}{ }^{ \pm} / B^{ \pm}$production cross section with the ATLAS detector at $\sqrt{S}_{\mathrm{s}}=8 \mathrm{TeV}$, arXiv:1912.02672 (2019), submitted to Phys. Rev. D.

[3] ATLAS Collaboration, "Study of the rare decays of $B_{s}{ }^{0}$ and $B^{0}$ mesons into muon pairs using data collected during 2015 and 2016 with the ATLAS detector," JHEP 04 (2019) 098, arXiv:1812.03017 [hep-ex].

[4] ATLAS Collaboration, "Study of the rare decays of $B_{s}{ }^{0}$ and $B^{0}$ mesons into muon pairs using data collected during the LHC Run 1 with the ATLAS detector," Eur. Phys. J. C 76 (2016) 513, arXiv:1604.04263 [hep-ex]. 